\title{
Waves in active matter: The transition from ballistic to diffusive behavior
}

\author{
A. R. Dulaney (1) and J. F. Brady* \\ Division of Chemistry and Chemical Engineering, California Institute of Technology, Pasadena, California 91125, USA
}

(Received 13 February 2020; accepted 23 April 2020; published 18 May 2020)

\begin{abstract}
We highlight the unique wavelike character observed in the relaxation dynamics of active systems via a Smoluchowski based theoretical framework and Brownian dynamic simulations. Persistent swimming motion results in wavelike dynamics until the advective swim displacements become sufficiently uncorrelated, at which point the motion becomes a random walk process characterized by a swim diffusivity, $D^{\text {swim }}=U_{0}^{2} \tau_{R} /[d(d-1)]$, dependent on the speed of swimming $U_{0}$, reorientation time $\tau_{R}$, and reorientation dimension $d$. This change in behavior is described by a telegraph equation, which governs the transition from ballistic wavelike motion to long-time diffusive motion. We study the relaxation of active Brownian particles from an instantaneous source, and provide an explanation for the nonmonotonicity observed in the intermediate scattering function. Using our simple kinetic model we provide the density distribution for the diffusion of active particles released from a line source as a function of time, position, and the ratio of the activity to thermal energy. We extend our analysis to include the effects of an external field on particle spreading to further understand how reorientation events in the active force vector affect relaxation. The strength of the applied external field is shown to be inversely proportional to the decay of the wavelike structure. Our theoretical description for the evolution of the number density agrees with Brownian dynamic simulation data.
\end{abstract}

DOI: 10.1103/PhysRevE.101.052609

\section{INTRODUCTION}

Active systems have garnered significant attention for their interesting phenomena such as motility induced phase separation and spontaneous self-assembly [1,2]. While much work has been done to study the steady-state behavior of active systems, the time dependent nature of these systems and the interplay between their modes of relaxation have not been studied in as much detail. Foundational work related to this topic has been established [3-5], but with a focus on the intermediate scattering function (ISF) [6-9]. Here, we focus on the fundamental difference in the relaxation of active systems compared to their passive counterparts and how activity alters the characteristics of their short-time dynamics. The persistent swimming motion of active particles results in fundamentally different modes of relaxation, which leads to interesting phenomena not present in either purely advective or diffusive systems.

The initial relaxation response of a confined active system results in a ballistic explosion, as observed by Takatori et al. [5]. When active Janus particles are released from a trap they initially explode outward and eventually become diffusive after several reorientation times. The initial directed motion results in a shock wave in density that radiates outward from the trap until the persistent swimming motion becomes uncorrelated. This wave motion is a direct result of the swimming and is present in all active systems.

In simple passive colloidal systems, thermal diffusion is the only relaxation mode. However, for active colloidal particles there is an additional mechanism that originates from

*jfbrady@caltech.edu the activity. When probing times less than the timescale for reorientation of an active particle there is a directed and nonzero average for the active propulsive force. Since the reorientation process is stochastic, at times long compared to the reorientation timescale the active force has zero mean and the ballistic motion becomes diffusive and scales with the thermal energy plus the activity [10].

In this paper we bring to light the inherent wavelike behavior exhibited by active systems relaxing from perturbed states and provide a fundamental explanation for this phenomenon, as well as its mathematical origins. In Sec. II we provide a general theoretical framework for describing active relaxation. In Secs. III and IV we apply the theory for the release of active Brownian particles (ABPs) from an instantaneous source in the limit of strong activity and when the thermal energy scale becomes comparable to the activity, respectively. Then in Sec. $\mathrm{V}$ we characterize the observed active waves in the presence of an external orienting field, and predict further instances of wavelike behavior in active systems. Finally, in Sec. VI we discuss the criterion necessary to observe wavelike behavior and provide concluding remarks. Our analysis and characterization focus on dilute colloidal suspensions of ABPs that self-propel with an inherent swim speed $U_{0}$ with orientation $\boldsymbol{q}$ and reorient on a characteristic timescale $\tau_{R}$. Interparticle and hydrodynamic interactions in the suspension are neglected in this paper.

\section{THEORETICAL FRAMEWORK}

We define a simple model for ABPs using the Smoluchowski equation for the probability distribution of particle positions and orientations. Unlike with passive, isotropic particles, the probability must be considered in positional $(\boldsymbol{x})$ and 
orientational $(\boldsymbol{q})$ space at each instant in time:

$$
\frac{\partial P(\boldsymbol{x}, \boldsymbol{q}, t)}{\partial t}+\nabla \cdot \boldsymbol{j}^{T}+\nabla_{R} \cdot \boldsymbol{j}^{R}=0 .
$$

The translational and rotational fluxes are given by $\boldsymbol{j}^{T}=U_{0} \boldsymbol{q} P-D_{T} \nabla P$ and $\boldsymbol{j}^{R}=-D_{R} \nabla_{R} P$, respectively, where $\nabla_{R}=\boldsymbol{q} \times \nabla_{q}$ is the rotational gradient operator. The contributions to the translational flux are from the advective swimming motion $U_{0} \boldsymbol{q} P$ and from thermal diffusion, where the translational diffusivity is defined using the Stokes-EinsteinSutherland relationship $D_{T}=k_{B} T / \zeta$. The rotational flux is only composed of a diffusive piece with rotational diffusivity $D_{R}=1 / \tau_{R}$.

Analytic solutions to Eq. (1) are elusive, but insight into the governing phenomena and wavelike structure can be obtained by expanding the Smoluchowski equation in orientational moments [11,12]. The first two orientational moments of Eq. (1) are governed by the conservation equations $[13,14]$ :

$$
\begin{gathered}
\frac{\partial n}{\partial t}+\nabla \cdot \boldsymbol{j}_{n}=0, \\
\boldsymbol{j}_{n}=U_{0} \boldsymbol{m}-D_{T} \nabla n, \\
\frac{\partial \boldsymbol{m}}{\partial t}+\nabla \cdot \boldsymbol{j}_{m}+(d-1) D_{R} \boldsymbol{m}=0, \\
\boldsymbol{j}_{m}=U_{0} \boldsymbol{Q}+\frac{1}{d} U_{0} n \boldsymbol{I}-D_{T} \nabla \boldsymbol{m},
\end{gathered}
$$

where $n(\boldsymbol{x}, t) \equiv \int P(\boldsymbol{x}, \boldsymbol{q}, t) d \boldsymbol{q}$ is the number density, $\boldsymbol{m}(\boldsymbol{x}, t) \equiv \int \boldsymbol{q} P(\boldsymbol{x}, \boldsymbol{q}, t) d \boldsymbol{q}$ is the polar-order field, $\boldsymbol{Q}(\boldsymbol{x}, t) \equiv$ $\int(\boldsymbol{q} \boldsymbol{q}-\boldsymbol{I} / d) P(\boldsymbol{x}, \boldsymbol{q}, t) d \boldsymbol{q}$ is the nematic order field, and $d$ is the orientational dimensionality (i.e., 2 for planar reorientations). Equations (2) and (3) are left as an open set of coupled equations which depend on subsequent orientational moments of $P(\boldsymbol{x}, \boldsymbol{q}, t)$.

Intuitively we understand that translational Brownian motion only gives rise to diffusion, which implies that the wavelike character must result from activity. Therefore to isolate this facet of particle motion we first focus on the limit of high activity relative to thermal energy, or when $D_{T} \rightarrow 0$. As a first order approximation we truncate the moment expansion by assuming the nematic order is isotropic, i.e., $\boldsymbol{Q}(\boldsymbol{x}, t)=0$, thus closing the above equations. Combining Eqs. (2) and (3) gives rise to a telegraph equation:

$$
\frac{\partial^{2} n}{\partial t^{2}}+\frac{(d-1)}{\tau_{R}} \frac{\partial n}{\partial t}=\frac{1}{d} U_{0}^{2} \nabla^{2} n .
$$

For times short compared to the reorientation time $\tau_{R}$, Eq. (4) has a wavelike character with wave speed $c=U_{0} / \sqrt{d}$ (similar to that obtained by Sevilla and Castro-Villarreal [9]), but for times long compared to $\tau_{R}$ the behavior is diffusive with the swim diffusivity $D^{\text {swim }}=U_{0}^{2} \tau_{R} / d$. Equation (4) in this form is similar to the model created by Alharbi and Petrovskii for population dynamics [15]. Fundamentally, ABP dynamics exhibit both wavelike behavior at short times and diffusive behavior at long times; this behavior is general for all active systems.

\section{WAVES FROM AN INSTANTANEOUS SOURCE}

We consider the unsteady behavior of active systems from an instantaneous source. That is, we focus on observing how the density of an active system in an open domain relaxes from a localized point source disturbance back to a uniform distribution. The point source can be thought of as an instantaneous, local addition of particles to an empty domain or as a local addition of particles overtop a homogeneous background concentration of particles. This problem's historical relevance in the field of diffusion and its potential to further the understanding of bacterial film propagation on surfaces [16] make it a prime initial example for comparing relaxation in active and passive systems. For brevity we will only consider sources in two spatial dimensions as these are most experimentally relevant, but the following analyses are readily extensible to three spatial dimensions.

The Green's function for Eq. (4) in two dimensions with radial symmetry is

$$
n(r, t)=\frac{2 \theta[(t-r) / \sqrt{2}]}{\sqrt{t^{2}-r^{2}}} e^{-\frac{1}{2} t} \cosh \left(\frac{\sqrt{t^{2}-r^{2}}}{2}\right),
$$

where $\theta(x)$ is the Heaviside function, $r$ is the radial displacement from the source normalized by run length, $l=U_{0} \tau_{R}$, and $t$ is time normalized by $\tau_{R}$. The Green's function corresponds to the release of active particles from an instantaneous source with randomly distributed initial orientations. Number density as a function of radial displacement is shown in Fig. 1 at multiple time points. At short times, $t<\tau_{R}$, particles swim outward from the origin, which leads to a sharp peak in the density. When $t \sim \tau_{R}$, particles have reoriented, reducing the maximum in the density. A small fraction of particles have retained a purely outward pointing trajectory which pushes the leading edge of the density profile slightly in front of the peak. The long wake is a result of the wavelike character dominating in the short-time regime and is characteristic of symmetric wave propagation in two spatial dimensions [17]. As $t>\tau_{R}$ the wavefront diminishes further and the density profile more closely resembles that of a diffusive system at all points away from the leading edge. When $t \gg \tau_{R}$ the density profile looks diffusive (not shown) for all positions with a diffusion coefficient given by the swim diffusivity.

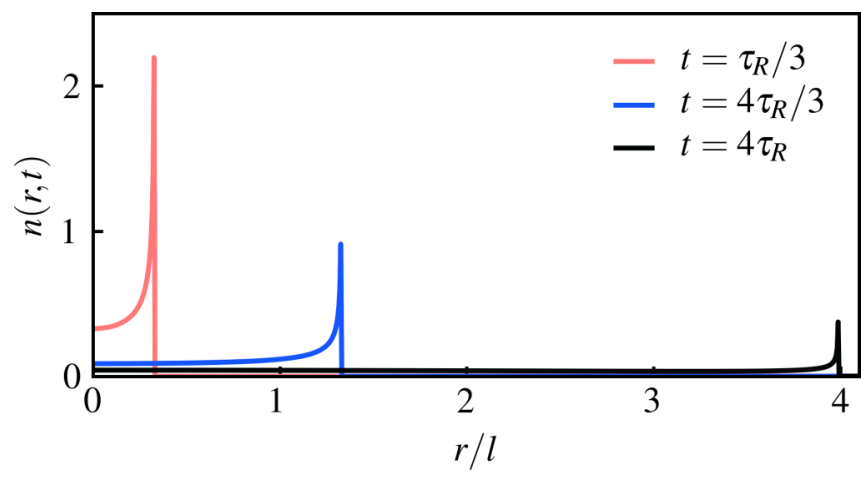

FIG. 1. Density as a function of radial position normalized by the run length, $l=U_{0} \tau_{R}$, in the limit of $D_{T} \rightarrow 0$ for the telegraph equation at times $t / \tau_{R}=1 / 3,4 / 3,4$. 


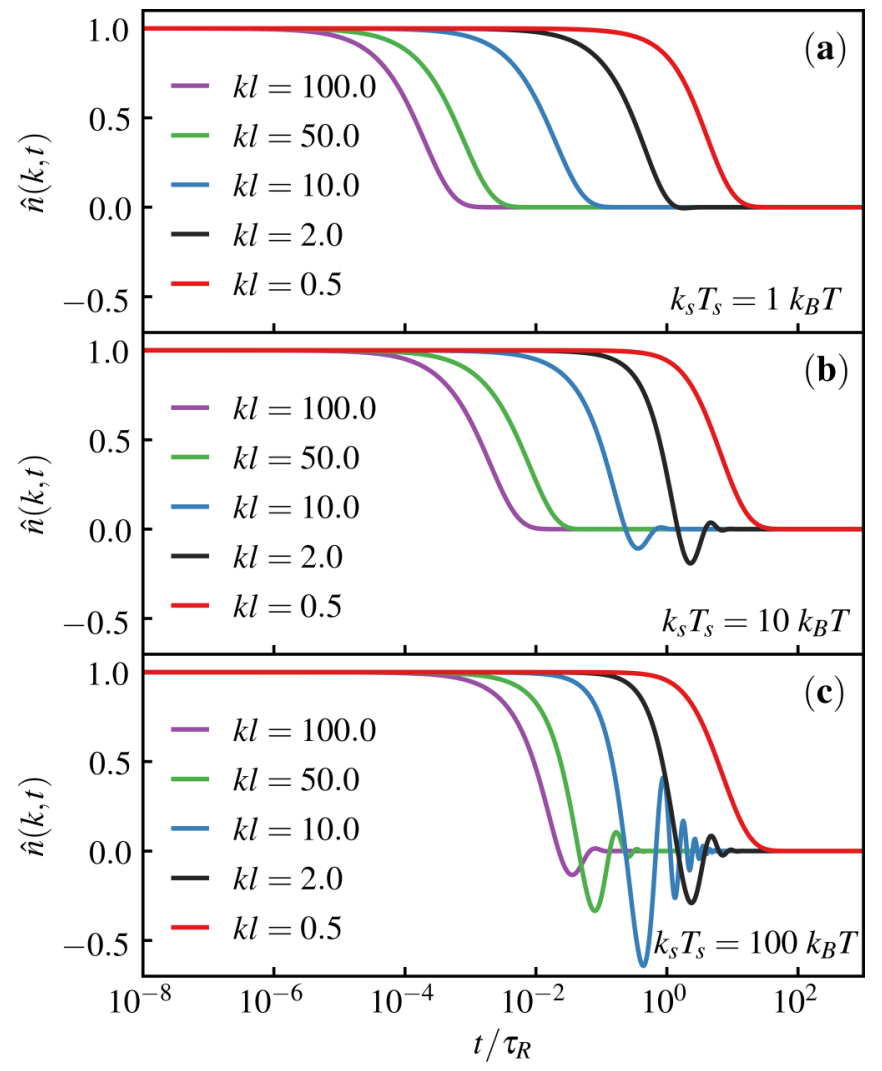

FIG. 2. Intermediate scattering function $\hat{n}(k, t)$ of ABPs for different values of dimensionless wave number, $k l$ (increasing from right to left), as a function of dimensionless time, $t / \tau_{R}$, for different levels of activity: (a) $k_{s} T_{s}=1 k_{B} T$, (b) $k_{s} T_{s}=10 k_{B} T$, and (c) $k_{s} T_{s}=$ $100 k_{B} T$ in two orientational dimensions.

Evidence of wavelike motion has been experimentally observed in systems of active Janus particles released from a two-dimensional, circular confining trap [5], but the experimental data imply a faster transition to diffusive dynamics. We believe the discrepancy between our simple model and these experimental data arises from the closure approximation that we have chosen, as evidenced by Fig. 3 in the following section. By neglecting the flux of polar order generated through nematic alignment, we have limited the diffusive character of the activity on longer timescales and thus prolonged the transition of the dynamics. This effect is exacerbated by the lack of thermal diffusion, which provides an additional mechanism through which the wavefront can relax, as will be seen in the following section. The telegraph equation shows the essential features of the ballistic to diffusive behavior [15], but it is not sufficient to quantitatively capture the transitional dynamics and is only strictly valid in the limit of high activity.

\section{THE EFFECTS OF DIFFUSION}

In this section, we revisit the relaxation of ABPs from an instantaneous source in the limit of finite activity to examine the interplay between thermal and active energy in the shorttime dynamics. We again start with Eqs. (2) and (3), but keep the translational diffusion terms. Scaling position and time by the particle run length and reorientation time, respectively,

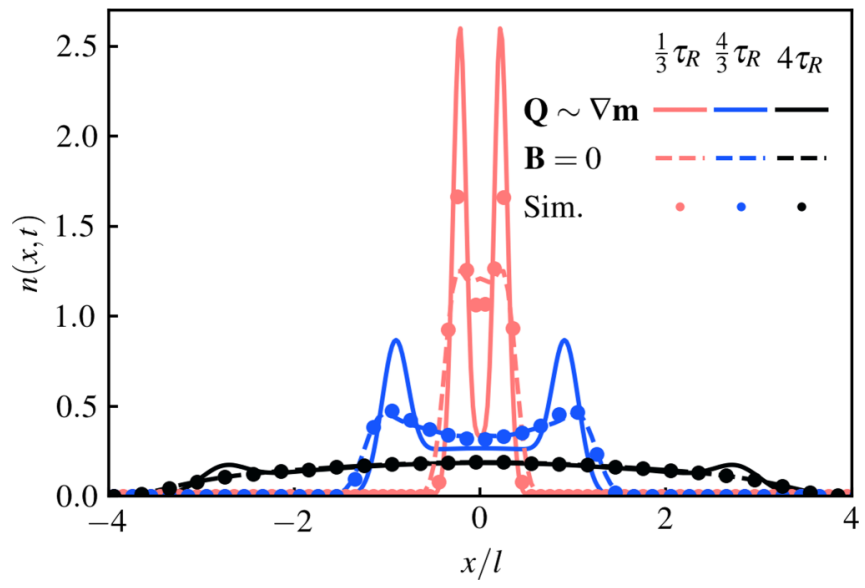

FIG. 3. Number density of particles as a function of position from an infinite line source with $k_{s} T_{s}=100 k_{B} T$. The solid lines, dashed lines, and symbols represent closures $\boldsymbol{Q}=\boldsymbol{A}: \nabla \boldsymbol{m}, \boldsymbol{B}=0$, and Brownian dynamic simulations, respectively. The red, blue, and black colors correspond to $t=\tau_{R} / 3,4 \tau_{R} / 3$, and $4 \tau_{R}$, respectively.

gives rise to a dimensionless parameter defined by the ratio of thermal energy, $k_{B} T$, to activity, $k_{s} T_{s} \equiv \zeta U_{0}^{2} \tau_{R} /[d(d-1)]$, for $d \geqslant 2$ [18]. As in Sec. II we formulate the set of scaled conservation equations as a single expression for the number density. The telegraph structure is still readily seen by Fourier transforming in position space, but now the first order time derivative and Laplacian terms have $k$-dependent coefficients:

$$
\frac{\partial^{2} \hat{n}}{\partial t^{2}}+f\left(\frac{k_{B} T}{k_{S} T_{S}}, k\right) \frac{\partial \hat{n}}{\partial t}=g\left(\frac{k_{B} T}{k_{S} T_{S}}, k\right) k^{2} \hat{n},
$$

where $\hat{n}(\boldsymbol{k}, t)$ is the transformed density and $\boldsymbol{k}$ is the dimensionless wave number. The spatially dependent coefficients are

$$
\begin{aligned}
& f=(d-1)+\frac{2 k^{2}}{d(d-1)}\left(\frac{k_{B} T}{k_{s} T_{s}}\right), \\
& g=\left[1+\frac{k_{B} T}{k_{s} T_{s}}+\frac{k^{2}}{d(d-1)^{2}}\left(\frac{k_{B} T}{k_{s} T_{s}}\right)^{2}\right] \frac{k^{2}}{d} .
\end{aligned}
$$

The transformed number density follows as

$$
\begin{aligned}
\hat{n}= & e^{-\left(\frac{(d-1)}{2}+\frac{k^{2}}{d(d-1)} \frac{k_{B} T}{k_{s} T_{s}}\right) t}\left\{\cosh \left(\frac{(d-1)}{2} \Gamma t\right)\right. \\
& \left.+\frac{1}{\Gamma} \sinh \left(\frac{(d-1)}{2} \Gamma t\right)\right\},
\end{aligned}
$$

where $\Gamma=\sqrt{1-4 k^{2} /\left[d(d-1)^{2}\right]}$. The spatially transformed density is equivalently the ISF as given by the Van Hove correlation [19]. Our analytic form of the ISF is similar to that obtained by Sevilla and Castro-Villarreal [9], though we do not utilize a memory function for the active motion. There has been broad interest in obtaining the ISF for active particles both experimentally [20-22] through differential dynamic microscopy and analytically [6-8]. Kurzthaler et al. numerically computed the ISF for anisotropic ABPs and anisotropic Brownian circle swimmers from an infinite expansion of the Smoluchowski equation in spherical harmonic functions [6-8], and Schwarz-Linek et al. solved the 
Smoluchowski equation using a series of Mathieu functions to compute the ISF of E. coli [22]. Our results show good agreement with the aforementioned methods while offering a more readily interpretable form. Figure 2 presents the ISF as a function of time for various wavelengths at three different levels of activity. When activity is comparable to thermal energy the ISF decays monotonically for all wave numbers, just as in a purely diffusive process [Fig. 2(a)]. As activity increases the decay of the ISF becomes nonmonotonic and has dampened oscillations at sufficiently large wave numbers [Figs. 2(b) and 2(c)]. For large $k$ the ISF decays monotonically and the onset of the decay begins sooner. When $k$ is large, diffusion dominates over the ballistic behavior of the active motion. As activity increases, the active contribution begins to dominate over thermal diffusion at shorter times and larger wave numbers, which extends the range of oscillations. These features agree with the results obtained by Kurzthaler et al. [6-8] for anisotropic and circle swimmers.

The oscillations in the structure factor correspond to time dependent density fluctuations, and are equivalent to those observed for the sinc function, which yields a dampened wave. As time increases towards the steady-state uniform distribution-approximately an order of magnitude beyond the reorientation timescale-the swimming motion becomes uncorrelated and each run length is a step in a random walk process, with a diffusivity governed by activity [23]. This transition corresponds to dampening of the oscillations observed in the decay of the ISF at small wave numbers. Mathematically, this transition is the point where the arguments of the hyperbolic functions in Eq. (8) become imaginary. That is, $\Gamma$ becomes imaginary when $k>(d-1) \sqrt{d} / 2$ and results in a critical dimensionless wave number $k^{\text {crit }} \simeq 0.71$ for our two-dimensional system. This transition from monotonic to oscillatory behavior as a function of wave number can be observed in Figs. 2(b) and 2(c) by the change in character from $k l=0.5$ (red) to $k l=2.0$ (black). We additionally computed the transition point from correlated wavelike to diffusive motion as a function of moment closure, the details of which are given in Appendix A.

A key benefit of the moment expansion method over a full numerical solution is the availability of an analytic form for the ISF. Asymptotic analyses of this analytic form provide insight into the physical nature of the observed phenomena beyond locating the wave transition. For large displacements $(k l \rightarrow 0)$ the density fluctuations decay as $\hat{n} \sim e^{-k^{2} D^{\text {eff }} t}$ with an effective diffusivity, $D^{\text {eff }}=D_{T}+2 D^{\text {swim }}$, where $D^{\text {swim }}$ is the diffusivity - as presented in Sec. II-that results when the swimming motion becomes uncorrelated. For small displacements $(k l \rightarrow \infty)$ the fluctuations are governed by dampened oscillations:

$$
\begin{aligned}
\hat{n}= & e^{-\left(\frac{(d-1)}{2}+\frac{k^{2}}{d(d-1)} \frac{k_{B} T}{k_{s} T_{s}}\right) t}\left\{\cos \left(\frac{k t}{\sqrt{d}}\right)\right. \\
& \left.+\frac{\sqrt{d}(d-1)}{2 k} \sin \left(\frac{k t}{\sqrt{d}}\right)\right\} .
\end{aligned}
$$

These oscillations in density capture the nondiffusive behavior witnessed by Takatori et al. [5].
In the short-time limit $t \rightarrow 0$, the mean-squared displacement (MSD) is $\left\langle x^{2}\right\rangle \sim 2 d D_{T} t+U_{0}^{2} t^{2}$, where the first term is the expected contribution from thermal motion and the second term arises from the ballistic swimming motion. This form of the MSD matches the results obtained by Ebbens et al. for their experimental catalytic swimmers [24]. The temporal scaling of each term explains the initial monotonicity in the decay of the ISF for times $t<\tau_{R}$ as $t>t^{2}$ and how this effect is reduced when activity is large. The ballistic scaling from activity is responsible for the "explosion" observed by Takatori et al. [5]. A full expression for the MSD is presented in Appendix C.

The real-space density profile for active particles diffusing from an instantaneous source can also be calculated. For the following analysis we consider the case of diffusion from an infinite line source for mathematical simplicity. Since this is effectively a one-dimensional problem, the long wakes present in Fig. 1 are no longer observed. The number density as a function of distance from the line source is shown in Fig. 3 at several instances in time for different closures to the moment hierarchy with an activity of $k_{s} T_{s}=100 k_{B} T$. While the first order closure to the moment hierarchy works very well for the ISF calculation, the large gradients in the initial density require a more sophisticated closure to obtain the real-space solution.

The transition from wavelike character is too slow with the simple $\boldsymbol{Q}=0$ closure, and requires an additional mode of relaxation. We do this by adding the effects of nematic order, which have thus far been neglected. In the presence of large spatial gradients $\boldsymbol{Q}$ is nonzero and therefore contributes to the speed of the relaxation of the wave. We rely on two different closures: assuming $\boldsymbol{Q}$ is slaved to the gradient of the polar order (solid lines) and assuming the third orientational moment $\boldsymbol{B}=\int[\boldsymbol{q} \boldsymbol{q} \boldsymbol{q}-\boldsymbol{\alpha} \cdot \boldsymbol{q} /(d+2)] P d \boldsymbol{q}$ is zero (dashed lines), where $\boldsymbol{\alpha}$ is the appropriate fourth order isotropic tensor. The first alternative closure, $\boldsymbol{Q} \sim \boldsymbol{A}$ : $\nabla \boldsymbol{m} \sim \nabla \boldsymbol{m}$, neglects $\boldsymbol{B}$ and higher order gradients of $\boldsymbol{Q}$, with constant tensor $\boldsymbol{A}=\boldsymbol{\alpha}-[(d+2) / d] \boldsymbol{I I}$ (see Appendix B for the exact closure) whereas the second closure only neglects $\boldsymbol{B}$ while retaining the effects of thermal diffusion on the nematic order.

For each closure, the density was calculated using a finite element method performed in FREEFEM ++ [25], and full Brownian dynamic (BD) simulations of ideal particles were performed to corroborate our results. The details of our simulations are outlined in Appendix D. Slaving $\boldsymbol{Q}$ to $\nabla \boldsymbol{m}$ (solid lines) correctly captures the short-time behavior of the system, but still fails to relax the wavefront quickly enough. Closing the expansion with $\boldsymbol{B}=0$ (dashed lines) matches the BD simulations well for $t \geqslant \tau_{R}$, but fails to capture the strong wavelike character at short times. Correctly capturing the behavior of the density for all times requires a more sophisticated closure for the expansion.

Snapshots of a characteristic simulation of ABPs released from a point source with $k_{s} T_{s}=100 k_{B} T$ are shown in Fig. 4 . The dense wave (yellow) at short times expands outward and spreads until the system reaches a uniform density (purple) after several $\tau_{R}$. This representation is evidence for the production of a relaxing wave that becomes diffusive, regardless of the dimensionality of the initial source. 

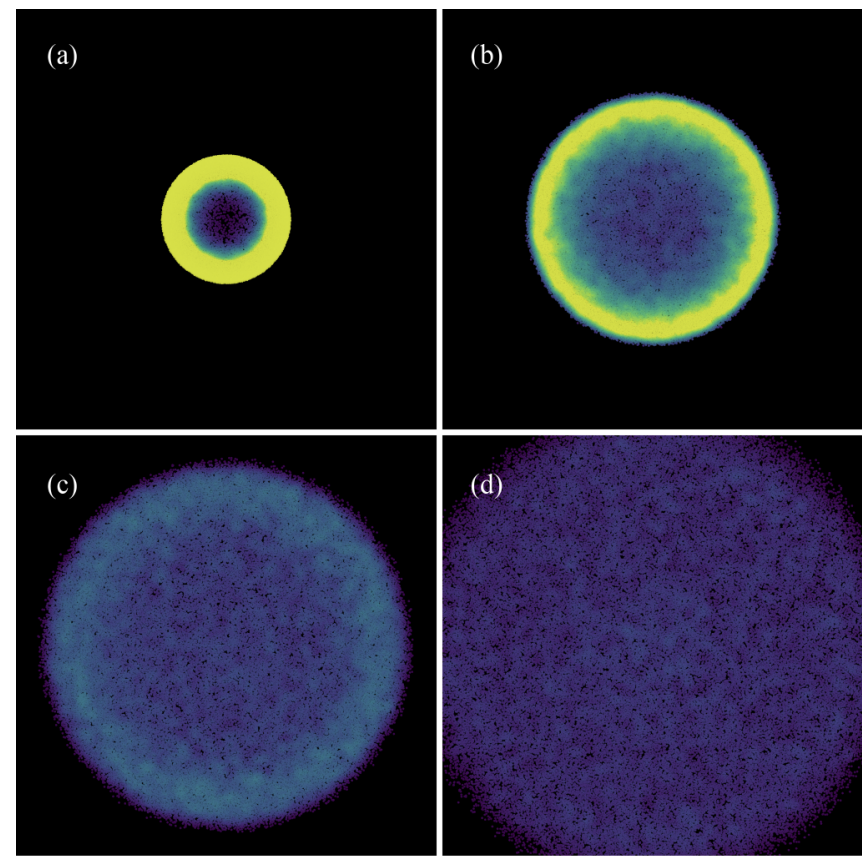

Density

Low High

FIG. 4. BD simulation snapshots for a system of ideal ABPs released from a two-dimensional point source with $k_{s} T_{s}=100 k_{B} T$ at (a) $t=\tau_{R}$, (b) $t=2 \tau_{R}$, (c) $t=3 \tau_{R}$, and (d) $t=4 \tau_{R}$. A dense wave of particles (yellow) can be seen spreading outward and diffusing until the system reaches a uniform density (purple).

Sokolov et al. observed similar wavelike behavior after cessation of rotation of a particle in a suspension of bacteria [26]. The rotating particle creates a stagnation zone wherein bacteria collect, which causes a large gradient in the radial density. After cessation of the particle, the bacteria explode outward in a wave, as indicated by Fig. 2(f) in [26] by the shifting peak in the density with time. We see in this instance, similar to the example of diffusion from an instantaneous source, that the wavelike behavior appears to result from large spatial disturbances in the density.

Thus far we have focused on suspensions of active Brownian particles released from instantaneous sources and have shown that the initial relaxation is ballistic and gets dampened out as the active motion becomes uncorrelated. As the motion decorrelates the relaxation resembles that of a diffusive process. In the following section we study the effects induced by the presence of an external orienting field and how this alters the relaxation behavior.

\section{EFFECTS OF AN EXTERNAL FIELD}

Consider the relaxation of active particles in the presence of an external orienting field. It is known that certain synthetic and living swimmers can be controlled through the application of external fields [27,28], but these works have focused on the steady-state regime [27], the strength of long-time response based on reorientation statistics [29], or how a strong perturbation to the field direction affects a single swimmer [28]. To study the relaxation we modify the rotational flux of Eq. (1) to

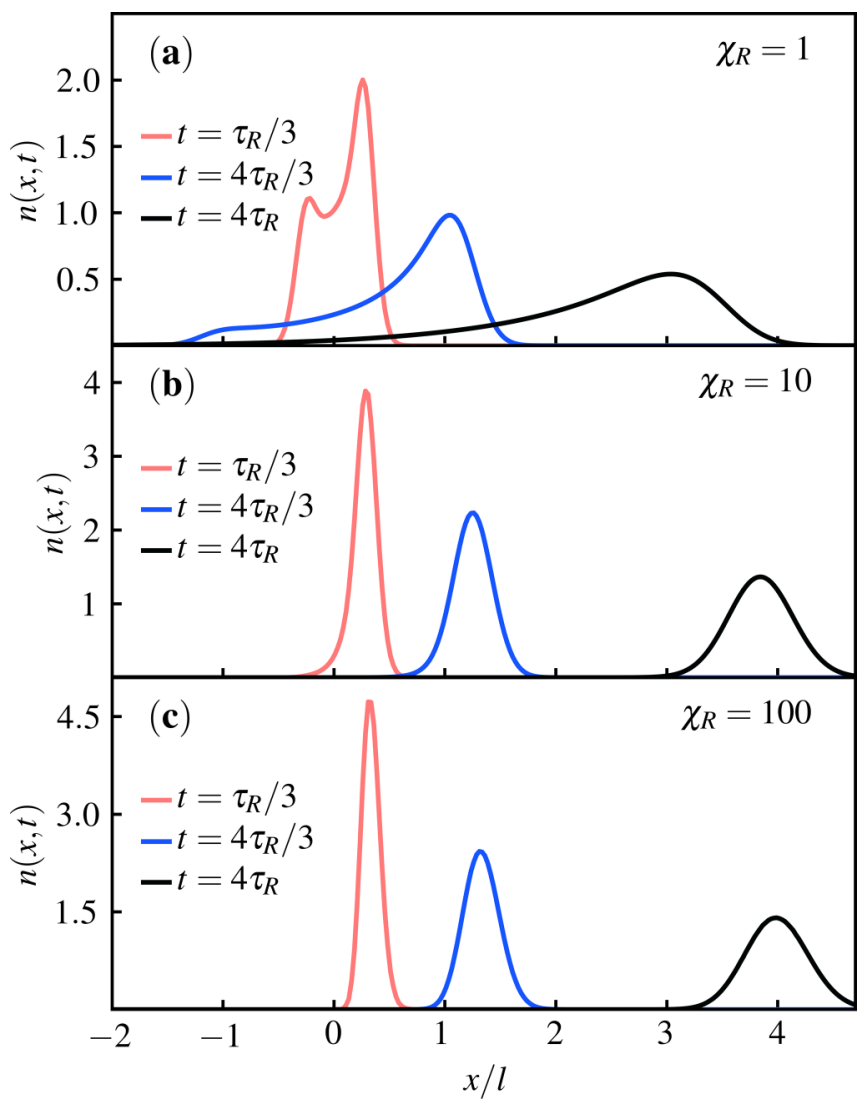

FIG. 5. The density profile as a function of displacement, at different moments in time with field strengths (a) $\chi_{R}=1$, (b) $\chi_{R}=$ 10 , and (c) $\chi_{R}=100$.

include the effects of an external orienting field. The rotational flux expression becomes

$$
\boldsymbol{j}_{R}=D_{R}\left(\chi_{R} \boldsymbol{q} \times \hat{\boldsymbol{H}} P-\nabla_{R} P\right),
$$

where $\hat{\boldsymbol{H}}$ is the unit vector in the field direction and $\chi_{R} \equiv \Omega_{c} \tau_{R}$ is the Langevin parameter with $\Omega_{c}$ being the magnitude of the angular velocity imposed by the field. Taking orientational moments results in the same expression for density conservation as before, but the expression for polar order becomes

$$
\begin{gathered}
\frac{\partial \boldsymbol{m}}{\partial t}+\nabla \cdot \boldsymbol{j}_{m}+\frac{(d-1)}{\tau_{R}} \boldsymbol{m}+\frac{\chi_{R}}{\tau_{R}} \boldsymbol{Q} \cdot \hat{\boldsymbol{H}} \\
-\frac{(d-1) \chi_{R}}{d \tau_{R}} n \hat{\boldsymbol{H}}=0, \\
\boldsymbol{j}_{m}=U_{0} \boldsymbol{Q}+\frac{1}{d} U_{0} n \boldsymbol{I}-D_{T} \nabla \boldsymbol{m} .
\end{gathered}
$$

We once again consider particles released from an infinite line source, with no initial polar order, but at time $t=0$ we apply an external field in the positive $x$ direction. The densities of particles as a function of position at times less than, comparable to, and greater than $\tau_{R}$ are presented in Fig. 5 for various values of the Langevin parameter for a highly active system, $k_{s} T_{s}=100 k_{B} T$. When $\chi_{R}$ is small [Fig. 5(a)] the density distribution looks similar to that presented in Fig. 3 when $t<\tau_{R}$, but with some asymmetry in the field direction. As time increases the peak moving opposite to 
the field relaxes faster while the peak moving in the field direction persists longer than it would in the absence of the field. As we increase the field strength to $\chi_{R}=10$ [Fig. 5(b)] the particles initially moving opposite to the field direction are quickly reoriented, resulting in a slight tailing of the density distribution, and when $t>\tau_{R}$ the particles have reoriented, thus reducing the tailing. Importantly, the spread in the density peak then is primarily from translational diffusion. When $\chi_{R}=100$ [Fig. 5(c)] the alignment effect is further enhanced and all particles are aligned in the field direction almost immediately after the field is turned on. This strong alignment prevents fluctuations in orientation from activity, resulting in a more persistent wavefront. The spread of the distribution is almost exclusively from thermal noise as evidenced by the mean and peak density speeds being equal to the swim speed. A strong orienting field reduces the swim diffusivity in the field direction as $\left\langle D_{\|}^{\text {swim }}\right\rangle \sim \mathcal{O}\left(\chi_{R}^{-3}\right)$ and as $\left\langle D_{\perp}^{\text {swim }}\right\rangle \sim$ $\mathcal{O}\left(\chi_{R}^{-2}\right)$ in the transverse direction, as shown by Takatori and Brady [27].

The persistence of the wave in the field direction and the dissipation of the wave in the opposite direction are better understood by calculating the mean-squared orientational displacement. The evolution for the average orientation is

$$
\frac{\partial}{\partial t}\langle\boldsymbol{q}\rangle=-\frac{(d-1)}{\tau_{R}}\langle\boldsymbol{q}\rangle-\frac{\chi_{R}}{\tau_{R}}\langle(\boldsymbol{q} \boldsymbol{q} \cdot \hat{\boldsymbol{H}}-\hat{\boldsymbol{H}})\rangle,
$$

where angled brackets represent an ensemble average. In the linear response regime (where $\chi_{R}$ is small) the rightmost term can be represented by $\langle(\boldsymbol{q} \boldsymbol{q} \cdot \hat{\boldsymbol{H}}-\hat{\boldsymbol{H}})\rangle_{0}=\int[(\boldsymbol{q q} \cdot \hat{\boldsymbol{H}}-$ $\hat{\boldsymbol{H}})] d \boldsymbol{q}=-(d-1) \hat{\boldsymbol{H}} / d$. From this

$$
\langle\boldsymbol{q}(t)\rangle=\frac{\chi_{R}}{d} \hat{\boldsymbol{H}}\left[1-e^{-(d-1) t / \tau_{R}}\right]+\boldsymbol{q}_{\mathbf{0}} e^{-(d-1) t / \tau_{R}},
$$

where $\boldsymbol{q}_{\mathbf{0}}=\langle\boldsymbol{q}(0)\rangle$. Multiplying Eq. (13) by $\boldsymbol{q}_{\mathbf{0}}$ and using $\left\langle(\boldsymbol{q}(t)-\boldsymbol{q}(0))^{2}\right\rangle=\left\langle\boldsymbol{q}^{2}(t)+\boldsymbol{q}^{2}(0)-2 \boldsymbol{q}(t) \cdot \boldsymbol{q}(0)\right\rangle$ in the limit of $t / \tau_{R} \ll 1$ gives a mean-squared orientational displacement of

$$
\left\langle(\boldsymbol{q}(t)-\boldsymbol{q}(0))^{2}\right\rangle \simeq 2(d-1)\left[1-\frac{\chi_{R}}{d}\langle\hat{\boldsymbol{H}} \cdot \boldsymbol{q}(0)\rangle\right] \frac{t}{\tau_{R}} .
$$

From Eqs. (13) and (14) we see mean-squared orientational displacement is greatly reduced in the field direction and enhanced in the opposite direction, resulting in the persistence and expedited dissipation of the two wavefronts, respectively. We can validate this result by computing the change to the average swim diffusivity $\left\langle D^{\text {swim }}\right\rangle \sim L_{\text {eff }}^{2} / \tau_{R}$, where $L_{\text {eff }} \sim\left(U_{0}-\right.$ $\langle u\rangle) \tau_{R}$ is the effective step size of a particle. The average velocity is given by $\langle\boldsymbol{u}\rangle=U_{0}\langle\boldsymbol{q}\rangle$, which is zero in the absence of an external field and is $\chi_{R} \hat{\boldsymbol{H}} / d$ with an external field in the linear response regime, as shown by taking the long-time limit of Eq. (13). This gives the change in effective step size as $\Delta L_{\text {eff }} \sim \chi_{R} U_{0} \tau_{R}$ and a scaling for the change in swim diffusivity as $\left\langle\Delta \boldsymbol{D}^{\text {swim }}\right\rangle \sim\left(U_{0}^{2} \tau_{R}\right) \chi_{R}^{2}$, which is in agreement with the predictions by Takatori and Brady [27]. Note that in the presence of an external orienting field the swim diffusivity is $\left\langle\boldsymbol{D}^{\text {swim }}\right\rangle \sim U_{0}^{2} \tau_{R}\left[1+\mathcal{O}\left(\chi_{R}^{2}\right)\right]$ and is anisotropic.

\section{CONCLUSIONS}

We have provided a model for active dynamics that provides insight into the wavelike behavior observed in active systems and have provided an explanation for how these dynamics relax. This motion and its transition to the steady-state diffusive behavior were shown through the relaxation of a dilute active system from an instantaneous source. The results were corroborated through $\mathrm{BD}$ simulations and comparison of the ISF with existing works [6-9]. At short times a strong wavelike character is present, and as the swimming motion becomes uncorrelated the overall motion becomes diffusive with an effective diffusivity given by the sum of the translational, $D_{T}$, and swim, $D^{\text {swim }}$, diffusivities and is described via the telegraph equation. The wavelike behavior observed for small displacements is supported by oscillations in density fluctuations in the system as predicted by the intermediate scattering function. We have calculated and correctly captured this behavior using a simple expansion of orientational moments with different closures: $\boldsymbol{Q}=0, \boldsymbol{Q} \sim \nabla \boldsymbol{m}$, and $\boldsymbol{B}=0$. While the majority of the relaxation results focus on diffusion from a line source, the methods shown readily extend to higher spatial dimensions.

The strength of an active wave can be maintained as shown by extension of the instantaneous source diffusion problem through the addition of an external orienting field. As the field strength increases, active constituents become unable to reorient, which is the primary mode of wave relaxation, thus increasing the life of the wave and allowing for greater displacements through more directed motion. The mean-squared orientational displacement of active particles in the presence of an orientating field compared to the unbiased case of free swimmers supports the claim in Takatori and Brady [27] that the persistence of the ballistic motion is primarily dependent on how correlated particle orientations are in time.

Given the observations presented we propose that the only criterion necessary for wavelike behavior is the existence of a mechanism through which the particle orientations can be recorrelated. This allows us to predict further instances wherein waves could be observed in active systems. For example, active suspensions near criticality should exhibit wavelike behavior as noncritical nuclei form and melt. The formation of noncritical nuclei results in large fluctuations in the density and their melting is analogous to the explosion of a "swimmer-crystal" observed by Takatori et al. [5] which, as previously discussed, clearly showcases a wave after release of the trap. Through our work we believe that waves are an inherent part of many active systems as they transition from a ballistic to diffusive motion.

\section{ACKNOWLEDGMENTS}

The authors would like to thank A. Omar, S. Mallory, and E. W. Burkholder for insightful discussions. This work was supported in part by the National Science Foundation under Grant No. CBET-1803662.

\section{APPENDIX A: WAVE TRANSITION}

Fourier transforming the conservation equations for the orientational moments of the probability distribution function $P(\boldsymbol{x}, \boldsymbol{q}, t)$ in space and subsequently Laplace transforming in time generates a system of algebraic equations. The system of equations can be rewritten as an explicit expression for $\hat{n}(\boldsymbol{k}, s)$ 
with the following structure:

$$
\hat{n}(\boldsymbol{k}, s)=\frac{1}{b_{0}+\frac{a_{1}}{b_{1}+\frac{a_{2}}{b_{2}+\cdots}}},
$$

which can be represented in continued fraction form as

$$
\hat{n}(\boldsymbol{k}, s)=\left[b_{0}+\boldsymbol{K}_{j=1}^{\infty} \frac{a_{j}}{b_{j}}\right]^{-1},
$$

where $s$ is the frequency. The terms $a_{j}$ and $b_{j}$ are given by the series

$$
\begin{aligned}
& a_{j}=\frac{(-1)^{j+1} j(d-3+j) U_{0}^{2} k^{2}}{(d-4+2 j)(d-2+2 j)}, \\
& b_{j}=\left[s+k^{2} D_{T}+j(d-2+j) D_{R}\right],
\end{aligned}
$$

where $a_{j}$ comes from the isotropic definition of the $(j+1)$ moment in the $j$ th moment equation and $b_{j}$ comes from the sink term on the $j$ th moment equation.

This expression for $\hat{n}(\boldsymbol{k}, s)$ is used to calculate the transition from wavelike to diffusive behavior. We demonstrate this by calculating the transition obtained from choosing the simplest moment closure of $\boldsymbol{Q}=0$. From the first two moments we obtain

$$
\hat{n}(\boldsymbol{k}, s)=\frac{1}{s+D_{T} k^{2}+\frac{\frac{1}{d} U_{0}^{2} k^{2}}{s+D_{T} k^{2}+(d-1) D_{R}}}
$$

for the transformed density. We then take the nondiffusive $\operatorname{limit}\left(\lim D_{T} \rightarrow 0\right)$, and nondimensionalize the wave number and frequency by $\overline{\boldsymbol{k}}=\boldsymbol{k} l$ and $\bar{s}=s \tau_{R}$, respectively. The number density expression then becomes

$$
\hat{n}=\frac{\tau_{R}[\bar{s}+(d-1)]}{\bar{s}^{2}+(d-1) \bar{s}+\frac{1}{d} \bar{k}^{2}},
$$

with poles given by

$$
\bar{s}^{2}+(d-1) \bar{s}+\frac{1}{d} \bar{k}^{2}=0
$$

The transition from wavelike to diffusive behavior occurs when the poles become imaginary, from which we have

$$
\bar{s}=-\frac{(d-1)}{2} \pm \frac{1}{2} \sqrt{(d-1)^{2}-\frac{4}{d} \bar{k}^{2}},
$$

and they become imaginary when $\bar{k}^{2}>d(d-1)^{2} / 4$, which matches the result we get from our analytic expression for the Fourier transformed number density.

\section{APPENDIX B: ALTERNATIVE CLOSURE FOR $Q$}

The conservation equation for the nematic order with a closure of $\boldsymbol{B}=0$ and neglecting high order spatial derivatives of $\boldsymbol{Q}$ gives

$$
\begin{aligned}
\frac{\partial \boldsymbol{Q}}{\partial t}+\nabla \cdot\left(\frac{U_{0}}{(d+2)}\left(\boldsymbol{\alpha}-\frac{(d+2)}{d} \boldsymbol{I I}\right) \cdot \boldsymbol{m}\right) \\
+\frac{2 d}{\tau_{R}} \boldsymbol{Q}=0
\end{aligned}
$$

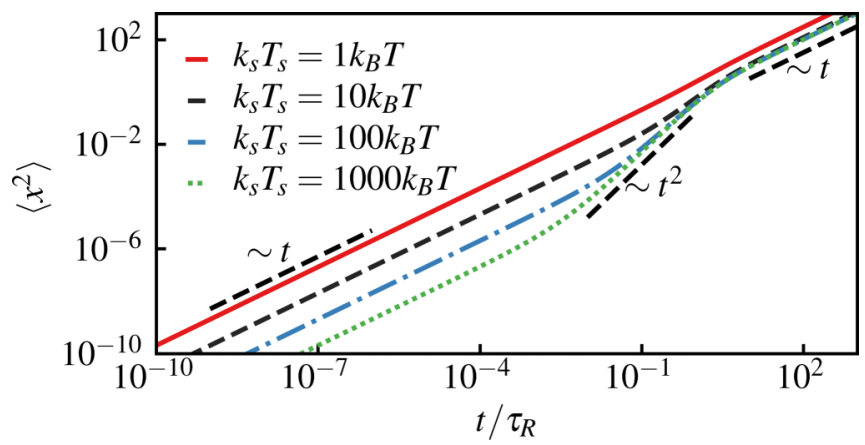

FIG. 6. The mean-squared displacement as a function of time normalized by the reorientation time at different levels of activity.

which can be rewritten as

$$
\boldsymbol{Q}=-\frac{U_{0}}{2 d(d+2) D_{R}}\left(1-e^{-2 d D_{R} t}\right) \nabla(\boldsymbol{A}: \boldsymbol{m}),
$$

where $\boldsymbol{A}=\boldsymbol{\alpha}-[(d+2) / d] \boldsymbol{I I}$ and $\boldsymbol{\alpha}$ is the appropriate isotropic fourth order tensor, as defined in the main text.

\section{APPENDIX C: MEAN-SQUARED DISPLACEMENT}

The MSD is given by

$$
\left\langle x^{2}\right\rangle=-\left.\nabla_{k} \nabla_{k} \hat{n}(\boldsymbol{k}, t)\right|_{k=0},
$$

for any instant in time. The full MSD expression with $\boldsymbol{Q}=0$ closure is

$$
\begin{aligned}
\left\langle x^{2}\right\rangle= & \frac{4 \alpha}{d(d-1)^{2}} e^{-\alpha}\left[\left(1+\frac{k_{B} T}{k_{s} T_{s}}\right) \cosh \alpha\right. \\
& \left.+\left(1-\frac{1}{\alpha}+\frac{k_{B} T}{k_{s} T_{s}}\right) \sinh \alpha\right]
\end{aligned}
$$

where $\alpha=\frac{(d-1)}{2} t$ and $t$ is normalized by the reorientation time $\tau_{R}$. A plot of the MSD is presented in Fig. 6 for various levels of activity. For $t \ll \tau_{R}$ the MSD scales linearly with time because thermal diffusion wins out over active motion. As time increases the MSD goes quadratically with time due to the persistent and directed active swimming. For $t>\tau_{R}$ the swimming motion becomes uncorrelated and the active particles become diffusive with an effective diffusivity which is a combination of the thermal diffusivity and swim diffusivity $D^{\text {swim. }}$.

\section{APPENDIX D: BROWNIAN DYNAMIC SIMULATIONS}

We simulate suspensions of ideal ABPs using the overdamped Langevin equations for translation and orientation:

$$
\begin{gathered}
0=-\zeta \mathbf{U}+\zeta \mathbf{U}_{0}+\mathbf{F}^{B}, \\
0=-\zeta_{R} \boldsymbol{\Omega}+\mathbf{L}^{R} .
\end{gathered}
$$

Here $\zeta$ is the hydrodynamic resistance coupling translational velocity to force, $\mathbf{U}$ is the translational velocity, $\zeta \boldsymbol{U}_{0}$ is the active-or swim-force [23], $\mathbf{F}^{B}$ is the random Brownian force, $\zeta_{R}$ is the hydrodynamic resistance coupling angular velocity to torque, $\boldsymbol{\Omega}$ is the angular velocity, and $\mathbf{L}^{R}$ is the 
reorientation torque. The Brownian force is modeled using the usual white-noise statistics with $\overline{\mathbf{F}^{B}}=0$ and $\overline{\mathbf{F}^{B}(0) \mathbf{F}^{B}(t)}=$
$2 k_{B} T \zeta \delta(t) \mathbf{I}$. The reorientation torque is modeled in similar fashion with $\overline{\mathbf{L}^{R}}=0$ and $\overline{\mathbf{L}^{R}(0) \mathbf{L}^{R}(t)}=2 \zeta_{R}^{2} \delta(t) \mathbf{I} / \tau_{R}$.
[1] C. Bechinger, R. Di Leonardo, H. Löwen, C. Reichhardt, G. Volpe, and G. Volpe, Rev. Mod. Phys. 88, 045006 (2016).

[2] S. A. Mallory, C. Valeriani, and A. Cacciuto, Ann. Rev. Phys. Chem. 69, 59 (2018).

[3] F. J. Sevilla and L. A. Gómez Nava, Phys. Rev. E 90, 022130 (2014).

[4] F. J. Sevilla and M. Sandoval, Phys. Rev. E 91, 052150 (2015).

[5] S. C. Takatori, R. De Dier, J. Vermant, and J. F. Brady, Nat. Commun. 7, 10694 (2016).

[6] C. Kurzthaler, S. Leitmann, and T. Franosch, Sci. Rep. 6, 36702 (2016).

[7] C. Kurzthaler and T. Franosch, Soft Matter 13, 6396 (2017).

[8] C. Kurzthaler, C. Devailly, J. Arlt, T. Franosch, W. C. K. Poon, V. A. Martinez, and A. T. Brown, Phys. Rev. Lett. 121, 078001 (2018).

[9] F. J. Sevilla and P. Castro-Villarreal, arXiv:1912.03425v1 (2019).

[10] S. C. Takatori and J. F. Brady, Current Opinion in Colloid Int. Sci. 21, 24 (2016).

[11] M. E. Cates and J. Tailleur, Europhys. Lett. 101, 20010 (2013).

[12] F. J. Sevilla, Phys. Rev. E 101, 022608 (2020).

[13] D. Saintillan and M. J. Shelley, in Complex Fluids in Biological Systems, edited by S. E. Spagnolie, Biological and Medical Physics, Biomedical Engineering (Springer, New York, 2015), Chap. 9, p. 449.

[14] W. Yan and J. F. Brady, J. Fluid Mech. 785, R1 (2015).

[15] W. Alharbi and S. Petrovskii, Mathematics 6, 59 (2018).
[16] H. Du, Z. Xu, M. Anyan, O. Kim, W. M. Leevy, J. D. Shrout, and M. Alber, Biophys. J. 103, 601 (2012).

[17] P. M. Morse and H. Feshbach, Methods of Theoretical Physics (McGraw-Hill, New York, 1953).

[18] S. C. Takatori and J. F. Brady, Phys. Rev. E 91, 032117 (2015).

[19] L. Van Hove, Phys. Rev. 95, 249 (1954).

[20] L. G. Wilson, V. A. Martinez, J. Schwarz-Linek, J. Tailleur, P. N. Pusey, W. C. K. Poon, and G. Bryant, Phys. Rev. Lett. 106, 018101 (2011).

[21] V. A. Martinez, R. Besseling, O. A. Croze, J. Tailleur, M. Reufer, J. Schwarz-Linek, L. G. Wilson, M. A. Bees, and W. C. Poon, Biophys. J. 103, 1637 (2012).

[22] J. Schwarz-Linek, J. Arlt, A. Jepson, A. Dawson, T. Vissers, D. Miroli, T. Pilizota, V. A. Martinez, and W. C. Poon, Colloids Surfaces B: Biointerfaces 137, 2 (2016).

[23] S. C. Takatori, W. Yan, and J. F. Brady, Phys. Rev. Lett. 113, 028103 (2014).

[24] S. Ebbens, R. A. L. Jones, A. J. Ryan, R. Golestanian, and J. R. Howse, Phys. Rev. E 82, 015304(R) (2010).

[25] F. Hecht, J. Numer. Math. 20, 251 (2012).

[26] A. Sokolov, L. D. Rubio, J. F. Brady, and I. S. Aranson, Nat. Commun. 9, 1322 (2018).

[27] S. C. Takatori and J. F. Brady, Soft Matter 10, 9433 (2014).

[28] W. Gao, X. Feng, A. Pei, Y. Gu, J. Li, and J. Wang, Nanoscale 5, 4696 (2013).

[29] B. Hancock and A. Baskaran, Phys. Rev. E 92, 052143 (2015). 